\title{
ENTRE LA IGUALDAD Y LA EFICIENCIA EN MATERIA SANITARIA
}

\author{
Alejandra Zúńiga Fajuri*
}

\begin{abstract}
Resumen: Este trabajo reflexiona en torno a los mecanismos de adjudicación de recursos sanitarios escasos propuestos por la "Economía de la Salud". Se analizan los modelos de costo-beneficio y el criterio QALY (Quality-Adjusted Life-Years) en tanto mecanismos de adjudicación que pretenden resolver los dilemas bioéticos relacionados con una distribución que parece priorizar resultados eficientes por sobre los resultados equitativos pero ineficientes.
\end{abstract}

Palabras-clave: salud, equidad, racionamiento

\section{BETWEEN EQUALITY AND EFFICIENCY IN MEDICAL CARE}

Abstract: This work considers the allocation methods of scarce medical resources budgeted for "health economics". Costbenefit models and the criteria QALY (Quality-Adjusted Life-Years) are analyzed as allocation methods that propose to resolve ethical dilemmas related to an economic distribution that seems to prioritize efficient results over more equitable, but inefficient, results.

Key words: health, equity, rationing

\section{ENTRE A IGUALDADE E A EFICIÊNCIA EM MATÉRIA SANITÁRIA}

Resumo: Este trabalho traz uma reflexão em torno dos mecanismos de adjudicação de recursos sanitários escassos propostos pela "Economia da Saúde". São analisados os modelos de custo-benefício e o critério QALY (Quality-Adjusted Life-Years) como mecanismos de adjudicação que pretendem resolver os dilemas bioéticos relacionados com uma distribuiçáo que parece priorizar resultados eficientes sobre os resultados eqüitativos, porém ineficientes.

Palavras-chave: saúde, eqüidade, racionamento

\footnotetext{
Doctora en Derecho. Profesora e investigadora de la Universidad de Valparaíso, Chile
}

Correspondencia: alejandra.zuniga@uv.cl 


\section{Introducción}

El presente trabajo tiene por objeto analizar, desde el punto de vista de la justicia distributiva, algunos de los más importantes sistemas de asignación de recursos sanitarios propuestos por la llamada "Economía de la Salud" para la solución de los problemas bioéticos derivados del racionamiento sanitario. Los dilemas asociados a los modos de limitación de recursos escasos en materia sanitaria poseen respuestas distintas, sea que se dé mayor o menor importancia a los criterios de equidad, por una parte, y de eficiencia, por otra.

¿Cómo se deben distribuir los recursos escasos entre los distintos pacientes? Los criterios desarrollados por la Economía de la Salud aspiran a establecer mecanismos objetivos e imparciales que permitan responder a las dificultades asociadas al racionamiento. Así, la incorporación de juicios de "eficiencia" a los clásicos mecanismos de distribución sanitaria sobre la base de las "necesidades" de los pacientes ha sido fuente de grandes polémicas y disputas -desde el ámbito de la bioética, la economía y la filosofía política- por los resultados inequitativos o discriminatorios que pudieran llegar a producir. En este artículo se intenta pasar revista a parte de esa interesante discusión.

\section{Eficiencia y racionamiento sanitario}

La Economía de la Salud se desarrolló a partir de los análisis de "coste-beneficio" ${ }^{\text {que dieron lugar al }}$ criterio Quality-Adjusted Life-Years, también conocido como QALY $^{2}$, y ampliamente utilizado para la distribución eficiente de recursos sanitarios. Este tipo de análisis se hizo conocido en 1987, a propósito del caso de Coby Howard, en Oregon, Estados Unidos.

El análisis costo-beneficio o costo-efectividad (Cost Effectiveness Analysis) es un tipo de evaluación económica completa para comparar distintas intervenciones de salud, en la que los efectos sobre los recursos se expresan en unidades monetarias y los efectos sobre la salud se expresan en unidades específicas no monetarias de efectividad, por ejemplo, número de vidas salvadas o de días libres de enfermedad. En los resultados de un análisis de costo-efectividad, los costos netos están relacionados con un solo indicador de efectividad. Por ello, es preciso que los principales resultados de todas las opciones relevantes puedan ser expresados mediante el mismo indicador de efectividad, aunque dicho indicador pueda tomar para cada opción una magnitud diferente (Gálvez Gonzáles AM. Guía metodológica para la evaluación económica en salud: Cuba 2003. Revista Cubana de Salud Pública 2004; 30(1): 37-54).

2 En castellano se le conoce como AVAC o "Años de vida ajustados a calidad".
El pequeño de siete años, al cual le fue diagnosticada leucemia, requería de un trasplante de médula que la legislatura de Oregon ya había decidido no costear para, en cambio, guardar esos recursos y satisfacer las necesidades médicas básicas de las personas de escasos recursos desprovistas de seguro médico. El caso llamó la atención de los medios y, aunque se inició una campaña para financiar la operación, el nińo murió antes de lograrlo.

En respuesta a ese dramático caso se propuso restaurar el financiamiento para ese tipo de trasplantes, pero John Kitzhaber -quien se transformaría en gobernador de Oregon- se opuso, argumentando que eso significaría dejar a muchos miles de personas sin seguro médico alguno, a cambio de ayudar a unos pocos, pues era financieramente imposible asistirlos a todos. En 1989, entonces, la legislatura de Oregon promulgó una reforma con dos cláusulas especiales: primero, la obligación para todos los empleadores privados de financiar seguros médicos para sus trabajadores y, segundo, la expansión del programa estatal Medicaid para cubrir a todas las personas del Estado que estuvieran bajo la línea de la pobreza (en ese momento, Medicaid cubría sólo al 42\% de las personas).

En los debates públicos en torno al plan de Oregon se reconoció que "no es económicamente racional ignorar el costo de oportunidad que significa proveer un cuidado sanitario de alto costo y bajo beneficio cuando existen tantas ganancias alternativas mejores que pueden entregarse con esos mismos recursos (...) (y) cuando ello significa el inutilizar esos recursos para alcanzar otras metas sanitarias o para satisfacer otras responsabilidades públicas importantes como la educación infantil"(1).

Luego, como el modelo de Oregon -que buscaba la expansión de la cobertura sanitaria- suponía pagar las nuevas prestaciones a través del racionamiento de los servicios, obtuvo innumerables críticas por la ética de racionar los recursos sólo para los pobres y el peligro de que, con el aumento del costo de los servicios, en poco tiempo la lista de éstos sería tan reducida que llegaría a ser insuficiente. Además, en un inicio los resultados del plan desafiaban el sentido común pues, por ejemplo, como se comprobó que el coste de una operación de apendicitis equivalía al de ciento cincuenta empastes dentales, la operación se situó en un lugar del ranking inferior al de estos últimos, a pesar que la vida de las personas dependía sólo de ésta. 
Para sus críticos, entonces, el plan de Oregon contenía serios defectos porque: "favorece muchos tratamientos relativamente menores por encima de tratamientos que salvan vidas, fomenta una concepción de la asistencia sanitaria de 'nosotros contra ellos', a través de su énfasis en los beneficios de los pobres y en las clasificaciones de prioridades, y carece de especificidad sobre las condiciones y tratamientos que serán y no serán cubiertos" (2). Además, el plan excluía a los discapacitados y a los ancianos al perder su "elegibilidad", por lo que estos grupos deberían continuar recibiendo los mismos beneficios que antes.

Por todo esto, la Comisión tuvo que abandonar el diseño del racionamiento basado exclusivamente en un análisis de "coste-beneficio" y dar cabida a un mecanismo más subjetivo que incorporara la valoración que los ciudadanos de Oregon daban a cada atención médica. Mediante la creación de una lista de servicios médicos, se redujeron los casi 10.000 procedimientos médicos a 709 condiciones médicas y sus tratamientos. Esta red de beneficios fue acordada por los propios ciudadanos del Estado, teniendo en cuenta el análisis de costo-beneficio de los tratamientos y las prioridades médicas de la propia comunidad. De este modo el "plan de racionamiento de Oregon, que se delineaba simultáneamente con el análisis 'costo-beneficio' y las preferencias de la población, representaba un inusual matrimonio entre las investigaciones sobre servicios sanitarios y la democracia deliberativa” (3).

La medida convencional de los QALYs -en la cual a cada "estado sanitario" le es asignado un peso numérico- es sólo uno de los métodos posibles de asignación de valor a los distintos "estatus de salud relacionados con la calidad de vida" (Health-related quality of life, $H R Q L)$. Dentro de los análisis de costo-efectividad, el criterio QALY es el método de selección más corriente, debido a su relativa simplicidad y fácil implementación. En su aplicación convencional, el peso asignado a cada estado de calidad regulado es multiplicado por el tiempo en ese estado (que puede ser discontinuo) y luego es sumado para calcular los años de vida ajustados a calidad. La ventaja de los QALYs, como medición de los resultados sanitarios, es que puede simultáneamente calcular las ganancias por la reducción de la morbilidad (calidad ganada) y la reducción de la mortalidad (cantidad ganada), e integrar ambos aspectos en una sola medición(4).
Para Alan Williams (a quien se atribuye la invención de este criterio) la esencia de los QALYs radica en darle valor de ' 1 ' a la esperanza de vida saludable de un año y en restarle ese mismo valor a la esperanza de vida no saludable de un año. Así, el valor preciso de la vida de una persona enferma disminuirá en la medida en que lo haga su calidad de vida. Si estar muerto tiene valor de ' 0 ', es en principio posible que un QALY tenga valor negativo, por ejemplo, cuando la calidad de vida de una persona se juzga como peor que estar muerto.

Un cuidado médico será beneficioso en la medida en que genere un resultado positivo de QALYs, y un cuidado médico eficiente será aquel en que el 'costopor-QALY' sea tan bajo como pueda ser. Un cuidado médico es prioritario cuando su 'coste-por-QALY' es bajo, y un cuidado es de baja prioridad cuando su 'coste-por-QALY' es alto. En la figura A se puede observar que, sin la intervención sanitaria, el estado de salud de una persona -relacionado con su calidad de vida-se deterioraría conforme con la curva decreciente y el individuo moriría en el punto 'Muerte 1'. Con la intervención, en cambio, esta persona se deterioraría más lentamente, viviría más y moriría en el punto 'Muerte 2'. El área entre las dos curvas es el número de QALYs ganados gracias a la intervención. Esta área puede dividirse en dos partes, A y B, donde la parte $\mathrm{A}$ es la cantidad de QALYs ganados debido a la calidad de las mejoras (por ejemplo, la calidad de vida ganada durante el tiempo en que la persona de todas maneras permanecería con vida), y la parte B es la cantidad de QALYs ganados debido a la cantidad de las mejoras (por ejemplo, la cantidad de vida extendida) (4).

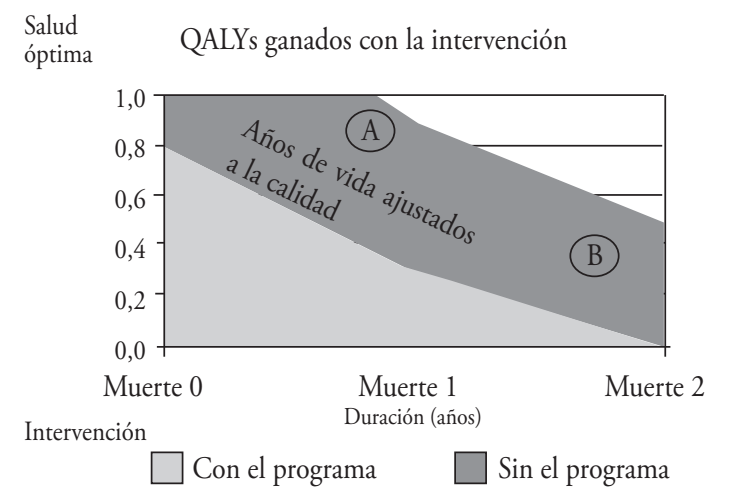

Fuente: Gold, 1996.

De este modo, sostiene Eric Nord, los QALYs funcionan básicamente como una medida desarrollada 
con el fin de resolver el problema de comparar "peras con manzanas" al momento de fijar prioridades en el cuidado sanitario. La idea es referirse a los diferentes resultados de salvar vidas, aumentar la expectativa de vida, el mejoramiento de distintos tipos de funciones y los distintos tipos de alivio de síntomas en la misma escala de valores. La característica clave del criterio de los QALYs es la valoración de la utilidad de los estados sanitarios donde, mientras más baja es la calidad de vida asociada a un estado sanitario más baja es la calificación de utilidad en esa escala. Por ejemplo, depender de muletas para caminar puede evaluarse con un puntaje de 0.9 , mientras que estar en una silla de ruedas se puede evaluar con un 0.8 . Si bien en la teoría económica el concepto de utilidad se define de muchas maneras distintas, Nord opta por un concepto pragmático que describe la utilidad de un estado sanitario como la 'bondad' de ese estado para quien se halla en él. Por bondad entiende el bienestar o buen estado asociado a esa condición.

\section{Los QALYs y la equidad}

En un entorno de permanente escasez de recursos, es crucial que el cuidado sanitario se entregue sin desperdiciarlo. Si bien el criterio QALY ha logrado combinar tanto el objetivo de alargar la vida junto con el de asegurar una cierta calidad de ésta, es un modelo de distribución con problemas de equidad que es fundamental evaluar. Como vimos, los QALYs tienen dos componentes: los "años de vida" y la "calidad de vida". El primer componente envuelve los conflictos en torno a la discriminación por edad, mientras que el segundo se relaciona con la discriminación por discapacidad, por sufrir enfermedades caras, por tener bajas posibilidades de recuperación o menor capacidad para beneficiarse con los tratamientos.

¿Cómo debe utilizarse este criterio de distribución? Los QALYs pueden usarse para determinar qué terapias rivales son mejores para un paciente particular o qué procedimiento es mejor para tratar una cierta condición. Claramente, aquella que genere más QALYs es la que deberá preferirse en un contexto de escasez de recursos. También pueden usarse para elegir, entre varios pacientes, cuáles deben ser tratados o qué condición debe ser prioritaria al momento de asignar recursos sanitarios. Este último es, según Harris, el uso que Alan Williams tenía en mente, pues se refiere precisamente al establecimiento de prioridades en el sistema sanitario en general. "Es este uso el que tendrá mayores efectos y más influencia al momento de buscar la eficiencia del sistema y es, precisamente, el uso que creo puede ser potencialmente peligroso y moralmente indefendible"(5).

¿Cuáles son los problemas de este modelo? Según Harris, toda la idea de los QALYs se basa en el supuesto general de que, dada la posibilidad de elección, cualquier persona racional preferirá una vida más breve, pero saludable, que una larga en condiciones de severa discapacidad o enfermedad. Si bien este supuesto permite deducir que el mejor tratamiento para esa persona será aquel que le permita una vida más larga con una mayor calidad -es decir, el tratamiento que rinda más QALYs-no permite concluir que los tratamientos con mayores resultados en QALYs sean preferibles cuando se trata de personas diferentes.

Esto es, "no podemos concluir que cuando la elección es entre tres años de enfermedad para mí o la inmediata muerte para ti, o un ańo de salud para ti y la inmediata muerte para otro, debemos necesariamente elegir la opción que te salve a ti y no a mí, pues ella produce mayor QALY de resultado. Por ejemplo, si tenemos 6 personas que podrían beneficiarse de un tratamiento y vivir una vida saludable por un ańo, y una sola que con ese mismo tratamiento podría vivir por 7 años, los QALYs nos recomendarían sacrificar a las 6 personas a favor de una sola"(5).

La ética de los QALY -que es la ética del utilitarismo- parece decirnos que el valor de toda vida humana es el mismo y que a los intereses de todos se les da igual peso. Pero en realidad a lo que se le da el mayor peso es al resultado QALY, el que, en la práctica, podría llevarnos a sacrificar los intereses de muchos en beneficio de unos pocos o a entregar todos los recursos disponibles para atender a los jóvenes pues producen más QALY. Como los años de vida que es posible esperar que una persona disfrute como consecuencia de un tratamiento disminuyen automáticamente cuanto mayor edad tiene el paciente, el criterio preferirá siempre al paciente más joven. Con él la pediatría acabaría consumiendo los mayores recursos y la geriatría, en cambio, quedaría al final de la lista. Más aún si se tiene en cuenta que en la actualidad, en los países desarrollados y en muchos en vías de desarrollo, el 60\% de los recursos se gastan en personas de más de 60 años (por ello, la aplicación del QALY haría estragos). Los defensores del criterio 
dirán que no hay nada de malo en gastar más en los pequeños que en los ancianos, pues la mayoría de la gente, teniendo que elegir, preferiría dar los recursos a los niños.

En el mismo sentido, se sostiene que los QALYs pueden discriminar por razón de sexo o de raza, pues si una condición sanitaria tiene mayor prioridad cuando el costo-por-QALY resulta ser más bajo y menor prioridad si resulta ser más alto, entonces las personas que sufran de condiciones más baratas tendrían siempre prioridad sobre aquellas personas víctimas de enfermedades más costosas. Esto no sólo envuelve un patrón de desventaja sistemática para ciertos grupos de pacientes, sino que también una posible preferencia permanente por la sobrevivencia de algunas personas en perjuicio de otras. Supongamos que las estadísticas médicas revelan que los asiáticos resisten mejor que otros después de cierta operación o de la aplicación de un determinado tratamiento, o que una condición particular, que tiene muy mal pronóstico en términos de QALY, afecta sólo a los judíos o a los homosexuales. Ese tipo de estadísticas, en una sociedad que se rige por los QALYs, podría significar el establecimiento de una discriminación persistente en contra de ciertos grupos identificables en términos raciales, de género, preferencia sexual, etc. $(5)^{3}$.

Siguiendo a John Rawls, una sociedad debe ser juzgada éticamente por como trata a sus miembros menos afortunados o peor situados. Una definición natural de los "peor situados" en relación con el cuidado sanitario es la de aquellos que carecen de seguro médico o de otros medios para satisfacer sus necesidades sanitarias(1). El criterio QALY parece empeorar la situación de los que ya están mal desde el punto de vista de la enfermedad, ya que si tuviéramos que elegir entre una persona discapacitada y otra sin ningún tipo de discapacidad como candidato a, por ejemplo, un trasplante, y suponiendo que el factor invalidez disminuye los éxitos del trasplante, se elegirá siempre a quien ya está en una mejor condición médica.

Finalmente, los críticos sostienen que el mecanismo QALY discrimina también por razones económicas, lo que se hace evidente cuando se incorporan mediciones

"Por ejemplo, no se debería trasplantar a un gitano, según este criterio, pues sus condiciones sociales y familiares disminuyen considerablemente el éxito del trasplante" (Puyol Á. Justícia i Salut. Ėtica per al Racionament dels Recursos Sanitaris. Barcelona: Universitat Autònoma de Barcelona; 1999: 139-140). de eficiencia a los tratamientos sanitarios. Desde ya los pacientes con enfermedades menos costosas tendrían que ser atendidos antes que aquellos que han desarrollado enfermedades con tratamientos más caros, por lo que estos últimos tendrían que contratar un seguro privado, suponiendo que puedan costearlo. "Ante la misma necesidad médica, el criterio da prioridad a quien solicita el tratamiento más barato. Ante distinta necesidad médica, va a priorizar a la persona que esté más sana"(6).

En definitiva, concluye Harris, los QALYs atentan directamente contra el principio de equidad, es decir, contra aquel principio que nos obliga a "tratar a las personas como iguales"; si se decide entregar a un individuo el tratamiento que le salve la vida debido a que tiene una mejor calidad de vida, o porque tiene más personas a su cargo o más amigos, o porque se considera que su vida es más "valiosa", se estaría tratando a algunos como más importantes que otros, lo que claramente viola el principio de equidad.

Cuando no hay recursos para salvarlos a todos, debemos elegir quiénes no serán salvados de un modo que no parezca una preferencia injusta. "Debemos tener muy claro que la obligación de salvar tantas vidas como sea posible no es la obligación de salvar tantas como podamos al menor costo posible"(5). Es por ello que estos autores prefieren que la selección de pacientes se haga por medio de mecanismos "imparciales" relacionados con el azar, como el criterio de hacer fila o el de la lotería. El uso de mecanismos impersonales como estos estaría justificado algunas veces por consideraciones de igualdad y oportunidad justa. Si los recursos sociales son escasos y no divisibles en porciones, y si no existen mayores disparidades en la utilidad médica para los pacientes (particularmente cuando la selección determina la vida o la muerte), entonces las consideraciones de oportunidad justa, de igual respeto e igual evaluación de las vidas podría justificar la práctica de hacer filas o una lotería.

Sin embargo, siguiendo a Calabresi y Bobbitt, considero que, al tratar del mismo modo a todas las personas, estos criterios encarnan la más simple e inocente concepción de la igualdad, pues "la lotería pura inevitablemente enfoca la indeterminación de primer orden creada por la escasez, lo que tiene el mismo efecto que valorar las distintas vidas, pues enfatiza nuestra incapacidad para defender como absolutos derechos como, por ejemplo, el derecho a la vida"(7). Dicho 
de otro modo, esta forma de racionamiento supone abdicar moralmente de las decisiones distributivas más importantes y trágicas, dejando al azar su resolución, aun cuando sea posible justificar moralmente la elección de unos pacientes por sobre otros, como veremos intenta hacer el utilitarismo.

\section{Una defensa de los QALYs}

Ya que resulta necesario sopesar los criterios de eficiencia con consideraciones de equidad, economistas como Nord y Williams han explorado mecanismos de armonización de estos indicadores. En esta misma línea, Mckie, Richardson, Singer y Kuhse desarrollan una defensa de los QALYs como parte de un sistema de cálculo utilitarista que puede, si es bien entendida, responder a las acusaciones de "injusticia" que he revisado.

En primer lugar los autores se preguntan: ¿cuánto debe gastar una sociedad para salvar una vida humana? Es necesario acordar qué queremos decir con gastar adecuadamente los recursos. ¿¿Significa que debemos distribuirlos lo más equitativamente posible, de manera que todos tengan acceso a la misma cantidad de bienes? ¿O alcanzar con ellos la mayor cantidad de beneficios? Al momento de acordar o definir un objetivo final de distribución es necesario, entonces, ponerse de acuerdo sobre qué tiene más valor para nosotros.

Sobre la acusación que sostiene que el criterio de maximización QALY discrimina a las personas de mayor edad, los autores responden que puede estar justificado dar mayor prioridad o peso "a los menores QALYs" que poseen las personas mayores si ello redunda en un mayor beneficio social. Es necesario tener presente la relación entre los QALYs y la teoría de la justicia del utilitarismo, en el sentido de que el criterio QALY es sólo uno de los componentes de la gran empresa de promover el "bienestar social", por lo que puede ser subordinado al objetivo general de maximizar la utilidad de la comunidad en su conjunto.

Entonces, sí existe un principio general sobre el cual justificar que se dé prioridad, en ciertas circunstancias, a las personas mayores o a otros grupos sociales: el principio de maximización de la utilidad social. Cualquier teoría sobre la distribución de recursos debe subordinarse al objetivo mayor de promover el bien de la comunidad, cuestión que resulta obvia cuando nos percatamos que muchos de los recursos que podríamos utilizar en salud se desvían hacia otras áreas sociales como la educación, el transporte, la vivienda, etc.(8).

Otra crítica al criterio QALY dice relación con la extensión de la vida. ¿Cuándo se debe gastar en recursos para alargar la vida de las personas? ¿En qué circunstancia se está ante una negación de recursos injustificada y, por ende, discriminatoria? Harris sugiere dejar este tipo de decisiones a los propios pacientes, pues sólo ellos pueden saber realmente lo importante que es seguir viviendo. Esto parece muy laudable pero, evidentemente, se trata de una posición que sólo puede aplicarse cuando no se está ante la competición por recursos escasos. Para McKie, Richardson, Singer y Kuhse resulta evidente que, cuando son varios los pacientes que quieren prolongar su vida y no hay fondos suficientes para todos, la tesis de Harris no nos sirve, ya que todos querrán salvar sus propias vidas. El problema de Harris, entonces, es que no entrega herramientas para decidir cómo y dónde fijar el umbral de atención(8).

Una posible solución a este dilema estaría contenida en la relación anunciada entre los QALYs y la tesis del utilitarismo. Si vemos el criterio QALY como una aplicación del utilitarismo en el área sanitaria, podremos aceptar que el umbral de atención estará fijado por el ideal de aumentar el bienestar social. De este modo, a veces lo mejor podrá ser posponer la muerte de una sola persona mientras que otras veces lo adecuado será posponer la de varias, dependiendo de las circunstancias del caso, es decir, de qué alternativa maximiza la utilidad general.

Si posponer la muerte del mayor número de individuos sólo supone una ganancia de unas horas, mientras que posponer la muerte de una sola persona puede significar una ganancia de varios años de vida, entonces probablemente lo mejor será entregar los recursos a esta última. "Que sea mejor asegurar más QALY para unas pocas personas o menos QALY para un mayor número dependerá de las circunstancias: en particular, de cuál opción produzca mayor bienestar. Esto muestra que el método QALY es sólo parte de todo el problema sobre la adjudicación de recursos. Es una parte importante pues permite, incuestionablemente, honrar dos importantes objetivos del cuidado sanitario: el mejoramiento de la calidad de vida y de la cantidad de vida”(8).

Finalmente, sobre la crítica relativa a que este sistema discriminaría a quienes sufren graves daños y enfermedades (pues la injusticia del criterio no sólo se reduciría 
a los "años de vida" -donde se discrimina a los mayores- sino también a la "calidad de vida" después de un tratamiento), los autores nos piden que imaginemos el siguiente ejemplo. Nora, que ha sido gravemente herida por un accidente de auto quedando parapléjica, estará confinada por el resto de su vida a una silla de ruedas y a sufrir fuertes dolores de espalda. Asumamos que dos años de vida en esas condiciones equivalen a un ańo de vida en condiciones de buena salud, de modo que los QALYs de Nora serán 0.5. Supongamos que, además, la expectativa de vida de Nora es de 40 años lo que, dada su mala condición de salud, equivale a 20 años de vida sana.

Ahora imaginemos que existe un tratamiento para el dolor de espalda de Nora que, si bien no le devolverá la capacidad de caminar ni aumentará su expectativa de vida, le quitaría por completo los dolores. En estas condiciones, su puntaje de QALYs aumentaría a 0.75 y su valor-vida crecería de 20 a 30 años. Comparemos la situación de Nora con la de Agnes quien, después del accidente de auto, no queda tan dañada. Si no recibe tratamiento más allá del servicio de atención de urgencia quedará lisiada, pero sin experimentar dolor o discapacidad como Nora a resultas de sus heridas. Esto le hace acreedora de un puntaje de 0.95 QALYs y si su expectativa de vida es de 40 ańos, como Nora, en su condición, ello equivaldría a 38 años de vida con calidad. Imaginemos ahora que hay un tratamiento para Agnes que podría eliminar completamente su cojera. Esto le devolvería el puntaje máximo de 1.0 y una cantidad/calidad de años de vida de 40 ańos.

Entonces, el problema se produce cuando asumimos que no podemos ayudar a Nora y a Agnes al mismo tiempo, y que tenemos que elegir a sólo una de ellas ¿ Es verdad que distribuir los recursos de modo de lograr el mayor número de QALYs dará siempre a Nora menos oportunidades de recibir el tratamiento que Agnes? Claramente no. Si bien entregar los recursos a Agnes implica como resultado un mayor número de QALYs (40 comparados con 30) sería un error pensar que este sería el resultado definitivo.

Si Nora recibe el tratamiento, su monto de QALYs postratamiento será de 30 y Agnes se mantendrá en su monto (sin tratamiento) de 38, lo cual sumaría un total de 68 QALYs. En cambio, si Agnes recibe el tratamiento, su monto posterior de QALYs sería 40, mientras que el de Nora se mantendría en 20, sin el tratamiento, lo que daría un total de 60 QALYs solamente. Esto muestra que, desde el punto de vista de la maximización de recursos, lo recomendable será dar prioridad a Nora, la persona más dañada por el accidente, pues ello producirá un monto total de QALYs superior. Esto muestra que no es verdad que este criterio discrimina sistemáticamente a las personas más severamente dañadas o enfermas, ya que todo dependerá del caso concreto $(8)$.

Lo anterior demuestra que la objeción de que el criterio de los QALYs se opone diametralmente al de distribución sanitaria conforme a la necesidad ${ }^{4}$ es injusta. Los autores sostienen que, si creemos que el fin de toda distribución debe ser la maximización de los QALYs, implícitamente estamos aceptando que el valor de un beneficio depende de su cantidad y no de quien lo reciba, a diferencia del principio de necesidad, para el cual el valor de un beneficio será diferente dependiendo de quien lo reciba, en particular, cuando lo reciben aquellos con grandes necesidades. El problema de esta tesis es cómo decidir cuando las necesidades de unos pueden superponerse a las de otros. Un modo de hacerlo sería explicando las necesidades en términos QALY. Por ejemplo, si Herbert tiene un monto menor de QALYs pretratamiento que Waldo, entonces es que está en gran necesidad, está sufriendo más debido a, por ejemplo, una grave discapacidad. Entendido de esta forma, la idea de comparar necesidades de diferentes personas no es más sospechosa que la de comparar los distintos niveles de QALY de las personas. Luego, si elegimos distribuir sobre la base de las necesidades, el objetivo será asegurar más QALYs para aquellos que tienen menos, incluso cuando ello signifique que los QALYs no sean maximizados. Los autores concluyen que, aunque el enfoque QALY es frecuentemente criticado como inequitativo, para el utilitarismo "distribuir recursos de modo que se maximice el bienestar humano, dando el mismo valor a los intereses de todas las personas en el proceso, es una expresión de nuestro rechazo a distribuir los recursos de manera sesgada, lo que refleja nuestro compromiso con la imparcialidad" (8). Aceptar un criterio como el de los QALYs es claramente preferible a "ningún criterio" de distribución, que es lo que finalmente plantearía Harris cuando deja la resolución del problema al azar. Decidir racionalmente, valorando la información que se posee, es una alternativa superior a la de la suerte.

\footnotetext{
Que podría entenderse comprendido en el "principio de diferencia" de Rawls, que distribuye los recursos en beneficio de los peor situados, a menos que una distribución alternativa les beneficie más.
} 
Adam Wagstaff, por su parte, tampoco cree que sea del todo cierto que el utilitarismo no muestra preocupación alguna por los problemas de distribución y equidad. Lo que ocurre es que el criterio QALY contiene su propio concepto de equidad sobre la base de que los QALYs de todos poseerían igual valor. Este principio implica que el resultado de la adjudicación de recursos por esta vía es automáticamente justo, independiente del grado de injusticia que envuelva y el tipo de persona que sea perjudicada(9).

Culyer ofrece una defensa aún más pragmática de la maximización sanitaria. Sugiere que, en efecto, es más equitativo discriminar a aquellos cuya capacidad de beneficiarse de los tratamientos médicos es limita$\mathrm{da}(10)$. Con todo, el problema es que precisamente la mejor capacidad de algunos para beneficiarse de los tratamientos puede deberse al simple hecho de que, por ser personas ricas, estén mejor alimentadas y tengan mejores conocimientos sobre las cuestiones sanitarias.

En fin, "si tenemos que elegir entre salvar la vida de Singer o la vida de Harris, deberemos salvar la que esperamos que se beneficie más de haber sido salvada. El hecho de que podamos tomar la decisión equivocada, y que nunca podamos saberlo, sólo es reflejo de nuestras humanas limitaciones. Pero afrontar la posibilidad de estar equivocados no debe impedirnos tomar las decisiones difíciles de la mejor forma que podamos, y no debe forzarnos a dejárselas a la suerte" (8).

Para lograr una distribución de recursos sanitarios que pretenda ser justa, tenemos que incorporar criterios de eficiencia que permitan adjudicar recursos de un modo que honre las exigencias de igualdad, responsabilidad y eficiencia de todo administrador de recursos escasos.

\section{Conclusión}

¿Juega la eficiencia algún papel en la evaluación de los estados sociales? Es un principio de racionalidad práctica sostener que es bueno alcanzar más de aquello que se considera valioso(11). La eficiencia es, sin duda, un elemento de la justicia social, de modo que todo principio de justicia distributiva debiera poseer alguna pretensión maximizadora.

Cuando se está ante decisiones trágicas, es decir, ante aquel tipo de elecciones distributivas en las que cualquier decisión afecta de manera sustancial la vida de las personas, resulta crucial fundamentar moralmente las elecciones finales por medio de criterios imparciales. La incorporación de instrumentos de análisis propios de la economía a los mecanismos de distribución sanitaria ha permitido justificar medidas que, de otro modo, habrían debido dejarse a la arbitrariedad personal del distribuidor público o al azar.

No cabe duda que el criterio QALY no permite resolver todos los casos difíciles, así como tampoco puede utilizarse sin incorporar otro tipo de consideraciones relacionadas con el principio de equidad y no discriminación. Sin embargo, la inclusión de mecanismos "eficientes" de adjudicación de recursos sanitarios escasos es un importante aporte a la solución de los dilemas bioéticos clásicos asociados al racionamiento de recursos sanitarios.

\section{Referencias}

1. Brock DW. Justice and the ADA: Does Prioritizing and Rationing Health Care Discriminate Against the Disabled? Social Philosophy and Policy 1995; 12 (2): 159-184.

2. Beauchamp T, Childress J. Principios de Ética Biomédica. Barcelona: Masson S.A.; 1999: 353-354.

3. Oberlander, Marmot \& Jacobs. Rationing Medical Care: Rhetoric and Reality in the Oregon Health Plan. Canadian Medical Association Journal 2001; 164(11): 1583-1587.

4. Gold MR, et al. Identifying and Valuing Outcomes. In: Gold MR, et al. (eds.) Cost-effectiveness in Health and Medicine. Oxford: Oxford University Press; 1996.

5. Harris J. QALYfying the Value of Life. Journal of Medical Ethics 1987; 13: 117-123.

6. Puyol Á. Justícia i Salut. Ėtica per al Racionament dels Recursos Sanitaris. Barcelona: Universitat Autònoma de Barcelona; 1999.

7. Calabresi G, Bobbitt Ph. Tragic Choice. New Cork, London: Norton \& Company; 1978.

8. McKie J, Richardson J, Singer P, Kuhse H. The Allocation of Health Care Resources. An Ethical Evaluation of the 'QALY' Approach. London: Dartmouth Publishing Company, Ashgate Publishing Limited; 1998. 
Entre la igualdad y la eficiencia en materia sanitaria - Alejandra Zúñiga Fajuri

9. Wagstaff A. QALY and the Equity-efficiency Trade-off. Journal of Health Economics 1999; 10: 21-41.

10. Culyer AJ. The Normative Economics of Health Care Finance and Provision. Oxford Review of Economic Policy 1989; 5(1):34-58.

11. Bayón JC. Justicia y eficiencia. En: Díaz E, Colomer JL. (eds.) Estado, Justicia, Derechos. Filosofía y Pensamiento. Madrid: Alianza; 2002.

Recibido: 12 de marzo de 2008

Aceptado: 13 de abril de 2008 